\title{
SAR AND TEMPERATURE DISTRIBUTIONS OF CYLINDRICAL ULTRASOUND TRANSDUCERS FOR INTRACAVITARY HYPERTHERMIA
}

\author{
Tzu-Chen Liang, Win-Li Lin, Wen-Chang Fan, Jia-Yush Yen, and Yung-Yaw Chen \\ Center for Biomed Eng, Depts of Mech Eng and Elec Eng, Nat'l Taiwan Univ, \\ Taipei, Taiwan. Fax: (886) 223940049
}

\begin{abstract}
The purpose of this paper is to examine the heating patterns when a cylindrical ultrasound transducer is employed for the intracavitary hyperthermia treatments. The present study employs a simulation program based on a simplified power deposition model for infinitely long cylindrical ultrasound transducers. The distribution of SAR ratio is used to determine the heating pattern for a set of given parameters. The parameters considered are the ultrasound attenuation in the tissue, the cavity size, and the transducer eccentricity. The results show that the ultrasound attenuation in the tissue, the cavity size and the transducer eccentricity are the most influential parameters for the distribution of SAR ratio. A low frequency transducer located in a large cavity can produce a much better penetration. The cavity size is the major parameter affecting the penetration depth for a small cavity size such as interstitial hyperthermia. The heating pattern can also be dramatically changed by the transducer eccentricity and radiating sector. The findings of the present study comprehend whether or not a tumor is treatable as well as select the optimal driving frequency, the appropriate cavity size and the eccentricity of a cylindrical transducer for a specific treatment.
\end{abstract}

Key Words: Cylindrical ultrasound transducer, Intracavitary hyperthermia, SAR ratio, Ultrasound attenuation, Transducer eccentricity, Cavity size.

\section{INTRODUCTION}

Diederich and Hynynen (1) examined the power deposition and temperature distribution for intracavitary ultrasound transducers with multi-element arrays. A complete intracavitary system with surface cooling water bolus has been constructed and is under clinical testing for the treatment of prostate (2), vaginal, and rectal tumors. Diederich and Burdette (3) evaluated a tubular multitransducer applicator designed for transurethral ultrasound thermotherapy of prostate tissue. Some investigators have also tried to design and evaluate the catheter-coupled tubular transducer devices along with the design and the feasibility of direct-coupled tubular transducer applicators suitable for simultaneous interstitial hyperthermia and radiotherapy (4). Cavity radius, transducer eccentricity (the shifting distance between the central axes of the transducer and the cavity), and ultrasound attenuation in the tissue are the parameters which potentially affect the ultrasound energy deposition in the tissue around a cavity during an intracavitary hyperthermia. The present study employs a power deposition model for cylindrical ultrasound transducers to examine the relationship between the parameters and the distributions of temperature and SAR ratio (specific absorption rate ratio) in the tissue around the cavity.

\section{Methods and Materials}

The deposition of ultrasound energy in the tissue around a cavity is modeled as a cylindrical transducer with uniform power that is emitted from the transducer face which penetrates into the tissue as shown in Figs. 1a-b. The radius of the transducer and the cavity is $R_{0}, R$, respectively. The power output per unit length from the face of the transducer is Q. The central axis of the transducer is shifted to a distance (d: transducer eccentricity) away from that of the cavity along the $x$-axis. The polar coordinates $(r, \theta)$ for a new coordinate system $o^{\prime}-x^{\prime}-y^{\prime}$ is employed to describe the ultrasound power deposition in the tissue. The ultrasonic beam is presumed to be collimated in the axial dimension but radially diverging from the face of the transducer. The deposition of ultrasonic intensity at each cylindrical shell, with respect to the transducer central axis, is simplified as a uniform distribution. Moreover the ultrasound power in the tissue is assumed to be exponentially attenuated according to the penetration depth of the ultrasound beam. A uniform attenuation of ultrasound is assumed for the entire tissue as well.

As the central axis of the transducer is coincident with that of the cavity (transducer eccentricity $(d)=0$ ), the ultrasound power deposition in the tissue is symmetrical to the central axis. The parameters for the power deposition are the ultrasound attenuation $(\mu)$ and the radius of the cavity (R). Hence, the ultrasound intensity on the cylindrical shell with radius $r$ in the tissue is 


$$
I(\mu, R ; r)=\frac{Q \cdot e^{-2 \mu(r-R)}}{2 \pi r}
$$

and the ultrasound intensity on the surface of the cavity is

$$
I(\mu, R ; R)=\frac{Q}{2 \pi R}
$$

where $\mu$ : ultrasound attenuation in the tissue; R: radius of the cavity; Q: ultrasound energy emitted from a unit length of the transducer surface.

Assume that the ultrasound intensities are not large enough to cause wave distortion and the attenuation and absorption coefficients of the tissue are equal (5). Then, we can obtain the ratio of SAR on the cylindrical shell with radius $r$ in the tissue to that on the surface of the cavity as

$$
\begin{aligned}
\operatorname{SR}(\mu, R ; r, R) & =\frac{\operatorname{SAR}(\mu, R ; r)}{\operatorname{SAR}(\mu, R ; R)} \\
& =\frac{I(\mu, R ; r)}{I(\mu, R ; R)}=\frac{R}{r} e^{-2 \mu(r-R)}
\end{aligned}
$$

Where $\operatorname{SR}(\mu, \mathrm{R} ; \mathrm{r}, \mathrm{R})$ : SAR ratio of the cylindrical shell with radius $r$ in the tissue to the surface of the cavity.

As the central axis of the transducer is shifted to a distance away from that of the cavity (transducer's eccentricity $(d) \neq 0$ ), the ultrasound power deposition in the tissue becomes two-dimensional. The power deposition for the point $(r, \theta)$ in the tissue is contributed by the ultrasound beam as shown in Fig. $1 \mathrm{~b}$. $S(\mathrm{R}, \mathrm{d} ; \mathrm{r}, \theta)$, the penetrating distance of the ultrasound beam into the tissue from the central axis of transducer to the point $(r, \theta)$, can be obtained by solving

$\mathrm{R}^{2}=\mathrm{d}^{2}+(\mathrm{r}-\mathrm{S})^{2}+2 \mathrm{~d}(\mathrm{r}-\mathrm{S}) \cos \theta$

The ultrasound intensity of the point $(r, \theta)$ in the tissue is

$$
\mathrm{I}(\mu, \mathrm{R}, \mathrm{d} ; \mathrm{r}, \theta)=\frac{\mathrm{Q} \cdot \mathrm{e}^{-2 \mu \mathrm{S}}}{2 \pi \mathrm{r}}
$$

and the intensity of the point $\left(\mathrm{R}-\mathrm{d}, 0^{\circ}\right)$ on the surface of the cavity is

$$
I\left(\mu, R, d ; R-d, 0^{\circ}\right)=\frac{Q}{2 \pi(R-d)}
$$

The SAR ratio for the point $(r, \theta)$ in the tissue to the point $(R-$ $\mathrm{d}, 0^{\circ}$ ) on the surface of the cavity is

$\operatorname{SR}\left(\mu, \mathrm{R}, \mathrm{d} ; \mathrm{r}, \theta ; \mathrm{R}-\mathrm{d}, 0^{\circ}\right)=\frac{\mathrm{R}-\mathrm{d}}{\mathrm{r}} \cdot \mathrm{e}^{-2 \mu \mathrm{S}}$

Where $\operatorname{SR}\left(\mu, \mathrm{R}, \mathrm{d} ; \mathrm{r}, \theta ; \mathrm{R}-\mathrm{d}, 0^{\circ}\right)$ : SAR ratio of a point on the cylindrical shell with radius $r$ in the tissue to the point $\left(R-d, 0^{\circ}\right)$ on the surface of the cavity.

\section{Temperature Solver}

To solve for the temperature distribution, Pennes' (6) steady-state bioheat transfer equation (BHTE) was used,

$$
-k \nabla^{2} \mathrm{~T}+\mathrm{Wcb}\left(\mathrm{T}-\mathrm{T}_{\mathrm{ar}}\right)=\mathrm{q}
$$

The thermal conductivity $(\mathrm{k})$ is $0.5\left(\mathrm{Wm}^{-10} \mathrm{C}^{-1}\right)$, the specific heat of blood $(\mathrm{cb})$ is $3770\left(\mathrm{Jkg}^{-1}{ }^{\circ} \mathrm{C}^{-1}\right)$, and the arterial temperature $\left(\mathrm{T}_{\mathrm{ar}}\right)$ is $37^{\circ} \mathrm{C}$. The absorbed ultrasonic power density $(q=2 \mu \mathrm{I})$ was obtained using the above procedure for a given power $(\mathrm{Q})$ output per unit length from the transducer. All attenuated ultrasonic energy was assumed to be absorbed by the tissue in the heating region.

\section{RESULTS AND DISCUSSION}

To examine the effect of cavity size and ultrasound attenuation on the distribution of SAR ratio in the tissue when a cylindrical ultrasound transducer is used, the cavity radius (R) varies from 5, 10, 15, 20, 25 to $\infty \mathrm{mm}$, and the ultrasound attenuation $(\mu)$ from $2.5,5,10,15,20$ to $30 \mathrm{~Np} / \mathrm{m}$. The central axis of the transducer is coincident with that of the cavity (transducer's eccentricity (d) $=0$ ), and hence the ultrasound power deposition in the tissue is one-dimensional. Figure 2 plots the depth of SAR ratio that equals to 0.5 based on equation (2.a). The figure shows that the depth of SAR ratio which equals to 0.5 is related to the attenuation and the size of the cavity. A larger cavity and/or a lower attenuation produces a larger depth of SAR ratio which equals to 0.5 . This finding suggests that a lower attenuation with a larger cavity has a better penetration. The results are presented in terms of tissues with different acoustic attenuation properties. 
Once the attenuation coefficient of the treatment tissue is known, an appropriate driving frequency can be obtained.

As the central axis of cylindrical transducer is shifted to a distance away from that of the cavity (transducer's eccentricity $(d) \neq 0$ ), the ultrasound power deposition in the tissue becomes two-dimensional. The ultrasound attenuation $(\mu)$, cavity radius (R), and transducer eccentricity (d) are the factors which potentially affect the distribution of SAR ratio. Figs. 3a-b display the two-dimensional distributions of SAR ratio based on equation (5) for an ultrasound attenuation of $2.5 \mathrm{~Np} / \mathrm{m}$, cavity radius of $20 \mathrm{~mm}$, and transducer radius of $5 \mathrm{~mm}$, while the transducer eccentricity (d) is 0 and $10 \mathrm{~mm}$, respectively. The figures indicate that the distribution of SAR ratio dramatically changes with the transducer eccentricity. Figs. 3c-d show the temperature distributions corresponding to the SAR ratio in Figs. 3a-b. The temperature of the cavity surface is set at $37^{\circ} \mathrm{C}$ and the maximum tissue temperature is maintained at $45^{\circ} \mathrm{C}$, while a uniform blood perfusion $\left(6 \mathrm{~kg} / \mathrm{m}^{3} / \mathrm{s}\right)$ is assumed for the entire simulated tissue. These figures display that a) the region of tissue close to the cylindrical transducer has a higher SAR ratio, b) the domain of SAR ratio higher than 0.5 converges to a smaller region and the gradient of SAR ratio becomes sharper as the transducer is shifted closer to the cavity surface, c) while the region with high SAR and temperature shifts to the corner as shown in Figs. 3b \& 3d. The transducer eccentricity provides a directional heating and the region of SAR ratio higher than 0.5 is controlled by the eccentricity.

\section{CONCLUSION}

The study shows that: 1) the cavity size, transducer eccentricity, ultrasound attenuation in the tissue are the most influential factors for the SAR ratio distributions, (or the heating patterns); 2) high driving frequencies should be used for heating the region close to the cavity surface, and low frequencies for deeper and larger regions; 3 ) the SAR ratio distribution also depends on the cavity size; a larger cavity has a slower decay of SAR ratio and hence results in a better penetration; 4) the transducer eccentricity can create a high SAR ratio region close to the transducer.

\section{ACKNOWLEDGMENTS}

The authors would like to thank the National Science Council and the Department of Health of the Republic of China for partially supporting this research under Contract Nos. NSC 85-2213-E-002-033 and DOH 86-HR-635, respectively.

\section{REFERENCES}

[1]. Diederich, C.; Hynynen, K. The development of intracavitary ultrasonic applicators for hyperthermia: a theoretical and experimental study. Medical Physics 17: 626-634; 1990.

[2]. Fosmire, H; Hynynen, K.; Drach, G. W.; Stea, B.; Swift, P.; Cassady, J. R. Feasibility and toxicity of transrectal ultrasound hyperthermia in the treatment of locallyadvanced adenocarcinoma of the prostate. Int. J. Radiat. Oncol. Biol. Phys. 26:253-259; 1993.

[3]. Diederich, C. J.; Burdette, E. C. Transurethral ultrasound array for prostate thermal therapy: initial studies. IEEE T. Ultrasonics Ferroelec. and Freq. Contr. 43:1011-1022; 1996.

[4]. Diederich, C. J.; Khalil, I. S.; Stauffer, P. R.; Sneed, P. K.; and Phillips, T. L. Direct-coupled interstitial ultrasound applicators for simultaneous thermobrachytherapy: a feasibility study. Int. J. Hyperthermia 12:401-419; 1996.

[5]. Hynynen, K. Biophysics and technology of ultrasound hyperthermia. In: Gautherie M. ed. Method of External Hyperthermic Heating. Berlin: Springer-Verlag; 1990: 61-116.

[6]. Pennes, H. H. Analysis of tissue and arterial blood temperatures in the resting human forearm. J. Appl. Phys. 1: 93-122; 1948.

\section{FIGURE CAPTIONS}

Figure 1: Schematic diagram of the geometry for a cylindrical ultrasound transducer with a power $Q$ emitted per unit length is placed within a cavity; b) The central axis of the transducer is shifted to a distance (eccentricity (d)) away from that of the cavity along the $\mathrm{x}$-axis.

Figure 2: Depth of the SAR ratio equals to 0.5 for different sizes of cavity and ultrasound attenuations, when the transducer eccentricity (d) is equal to zero.

Figure 3: $(a-b)$ are the distributions of SAR ratio for a cylindrical transducer with $2.5 \mathrm{~Np} / \mathrm{m}$ attenuation $(\mu)$ and a $20 \mathrm{~mm}$ cavity radius $(\mathrm{R})$. The transducer eccentricity (d) is 0 and $10 \mathrm{~mm}$, respectively. Figs. (c-d) show the temperature distributions for Figs. (a-b), respectively. 

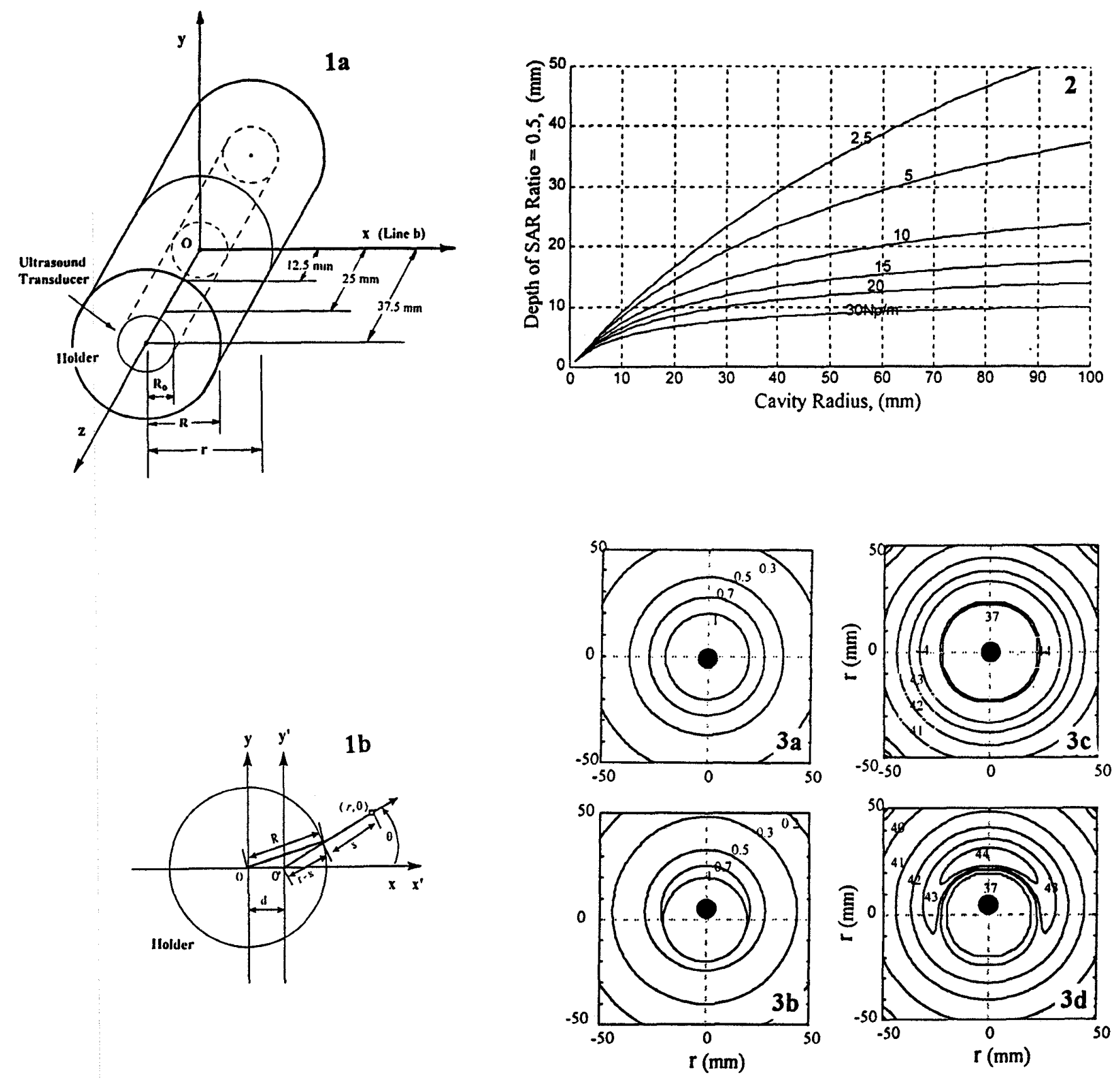